\title{
Using Astrometry to Deblend Microlensing Events
}

\author{
David M. Goldberg \\ Princeton University Observatory, Princeton, NJ 08544-1001 \\ email: goldberg@astro.princeton.edu
}

\begin{abstract}
We discuss the prospect of deblending microlensing events by observing astrometric shifts of the lensed stars. Since microlensing searches are generally performed in very crowded fields, it is expected that stars will be confusion limited rather than limited by photon statistics. By performing simulations of events in crowded fields, we find that if we assume a dark lens and that the lensed star obeys a power law luminosity function, $n(L) \propto L^{-\beta}$, over half the simulated events show a measurable astrometric shift. Our simulations included 20000 stars in a $256 \times 256$ Nyquist sampled CCD frame. For $\beta=2$, we found that $58 \%$ of the events were significantly blended $\left(F_{*} / F_{t o t} \leq 0.9\right)$, and of those, $73 \%$ had a large astrometric shift ( $\geq 0.5$ pixels). Likewise, for $\beta=3$, we found that $85 \%$ of the events were significantly blended, and that $85 \%$ of those had large shifts. Moreover, the shift is weakly correlated to the degree of blending, suggesting that it may be possible not only to detect the existence of a blend, but also to deblend events statistically using shift information.
\end{abstract}

Subject headings: methods: data analysis — gravitational lensing

\section{Introduction}

There has been significant discussion about blending in gravitational microlensing events (Di Stefano \& Esin 1995; Alard 1996b; Woźniak \& Paczyński 1997; Han 1997b). A blended microlensing event occurs when a lensed object cannot be resolved from other nearby objects in the field of view. In general, this can be caused by contributions from a bright lensing star, a binary companion to the lensed star, or a crowded field. Microlensing searches are typically conducted in very crowded fields in order to maximize the frequency of detections, and thus we shall primarily deal with the latter in this paper.

An observed star may be comprised of several contributing stars, and its total brightness can be determined by fitting a point spread function (PSF) to the light distribution on the 
CCD. The individidual brightnesses of the contributing stars, however, are unknown. We define the blending parameter, $f$, to be the ratio of the (unknown) brightness of the lensed star to the total measured brightness of the observed star. That is:

$$
f \equiv \frac{F_{s 0}}{\sum_{i} F_{i}}=\frac{F_{s 0}}{F_{0}}
$$

where $F_{s 0}$ is the baseline flux from the lensed star, $F_{i}$ are the fluxes from each contributing star in the observed image, and $F_{0}$ is the measured baseline flux of the image. Since only one star within the blend will be lensed, if the lensed star is magnified by some amount, $A$, then the total image will appear to be magnified by:

$$
A_{o b s}=1+f(A-1) \text {. }
$$

We draw a distinction between the actual magnification, $A$, and the observed magnification, $A_{o b s}$. The former is not known directly, and can only be inferred for a given value of $f$. The latter is given by the ratio of observed flux at some time to the baseline level.

If we make the simplifying assumption that the source and the lens are a point source and a point mass, respectively, and that the two are moving at constant speed relative to each other, then $A$ evolves in a very straightforward way (Paczyński 1986; for comprehensive reviews, see also Paczyński 1996; Alcock 1997):

$$
A(u)=\frac{u^{2}+2}{u\left(u^{2}+4\right)^{1 / 2}} ; \quad u^{2}(t)=u_{\min }^{2}+\left(\frac{t-t_{\max }}{t_{0}}\right)^{2}
$$

where $u$ is the angular distance of the lensed star from the lensing mass in terms of the Einstein radius, $u_{\min }$ is the impact parameter in the same units, $t_{\max }$ is the time of maximum brightness and $t_{0}$ is the characteristic time of the event:

$$
t_{0}=0.214 \mathrm{yr}\left(\frac{M}{M_{\odot}}\right)^{1 / 2}\left(\frac{D_{d}}{10 \mathrm{kpc}}\right)^{1 / 2}\left(1-\frac{D_{d}}{D_{s}}\right)^{1 / 2}\left(\frac{200 \mathrm{~km} \mathrm{~s}^{-1}}{V}\right) .
$$

where $M$ is the mass of the lens, $D_{d}$ is the distance from the observer to the lens, $D_{s}$ is the distance from the observer to the lensed star, and $V$ is the relative velocity of the two, projected into the plane of the lens.

Thus, a single lens microlensing event can be described by five parameters: $F_{0}, t_{0}$, $t_{\text {max }}, u_{\text {min }}$, and $f$. The total baseline flux, $F_{0}$ can be well measured with many observations of the star in the unlensed state, and symmetry shows that the time of peak amplification, $t_{\text {max }}$ is unaffected by blending. The three parameters, $f, t_{0}$, and $u_{\min }$ are not algebraically degenerate, but nevertheless form almost identical light curves. If the photometric measurements of the event are accurate enough, it may be possible to determine these 
three parameters by performing a best fit to the shape of the observed light curve. In fact, this has been successfully applied. Alard (1996b) shows OGLE \#5 to be strongly blended and the MACHO collaboration (Alcock et al. 1996) find 3 of the 9 observed events in the LMC to have significant blends. However, Woźniak and Paczyński (1997) show that for reasonable errors in the measurement of the light curve, $f \ll 1, t_{0}$, and $u_{\text {min }}$ form a degenerate set of models such that:

$$
f^{\prime}=f C ; \quad u_{\min }^{\prime}=u_{\min } C ; \quad t_{0}^{\prime}=t_{0} C^{-1}
$$

where $C$ is an arbitrary constant.

If we falsely assume a given event with blending fraction, $f$, to be unblended, then we may, in fact, be able to find a good fit of parameters in the least squares sense, but actually underestimate $t_{0}$ by a factor of $f^{-1}$. If we have a good model of the geometry and velocity distribution of the system, then this causes us to underestimate the mass by a factor of $f^{-2}$ (equation 4 ). For a large blending factor, this could result in mistaking a brown dwarf for a main sequence star.

There are some cases such as DUO \# 2 (Alard et al. 1995; Alard 1996a) OGLE \# 7 (Udalski et al. 1994a), and MACHO LMC \# 9 (Bennett et al. 1997) in which a binary system lenses a star (see Mao \& Di Stefano 1995 for details). In this case, the curves for different values of $f$ are distinguishable. Moreover, Witt \& Mao (1995) show that the true magnification of a lensed source within the caustics of a binary must be $\geq 3$, placing a strict upper limit on $f$ for a given value of $A_{o b s}^{\max }<3$. Indeed, substantial blending was found in all three cases, and may lead one to the suspicion that most single events toward the galactic bulge and the LMC are significantly blended as well.

Several methods have been suggested for detecting blends in single lens events. Some (Alard 1996b; Buchalter et al. 1996; Han 1997b) suggest that it may be possible to use the color shift of a blended event to determine the degree of blending. If the lensed star and the blended objects are of different colors, then over the course of the microlensing event, the color will shift from a weighted average all of the contributing stars to approximately the same color of the lensed star. While this measurement does not give an unambiguous measure of what the blending fraction is, it is nevertheless indicative that a blend does exist.

Due to the narrow distribution of colors in the galactic bulge (Udalski et al. 1993), it is difficult to detect significant color shifts in most events, but development of early warning alert systems in both the OGLE (Udalski et al. 1994b) and MACHO (Pratt et al. 1996) projects can permit hourly observations of an event in progress with small photometric errors, making it possible to detect a color shift. Using a detailed model, Buchalter et al. 
(1996) suggest that about $30 \%$ of bulge main sequence sources and about $7 \%$ of bulge giant sources will show a shift using these uncertainties. It should be noted that the detailed models of color shifts concentrate almost exclusively on blending by the lensing star, and suggests that crowding will cause a color shift in only $\sim 10 \%$ of events.

Han (1997b) proposes using the Hubble Space Telescope (HST) to do followup analysis of a lensing event. Since in the Galactic bulge there will typically only be $\sim 4$ stars within a seeing disk at or below 2 magnitudes above the detection limit, using the HST, one can determine the positions and colors of the individual component stars. By coupling this with the color information given by the chromatic shift, one can select the star that is being lensed, and determine $f$ and $t_{0}$.

However, there are several difficulties. First, the contribution of stars fainter than 2 magnitudes above the detection limit is non-negligible. The model used indicates that there are $\sim 20$ stars in a typical PSF down to $I=23$ magnitudes. This is crowded even at HST resolution. Since this limit constitutes a fractional contribution of 0.01 of the detection limit (at low $f$, the probability of a lensed event being observed goes as $f$ ), but increases the number of stars by an order of magnitude, these dim stars will constitute $\gtrsim 10 \%$ of the observed events. Moreover, the HST time involved is not inconsiderable ( $\sim 1 \mathrm{hr} /$ event).

Finally, Alcock et al. (1996) discuss performing a statistical correction to the timescales of observed events. By performing Monte Carlo simulations, they compute an estimate of the distribution of characteristic timescales, $t_{0}$, under the assumption that $f=1$ throughout. They can then multiply the observed timescales by a correction factor in order to determine the optical depth of the galactic halo to microlensing. In principle this will give a good overall estimate of the optical depth to microlensing, $\tau$, which is, after all, the ultimate goal of these searches. However, it does not give information about individual microlensing events. Furthermore, it requires a detailed model of the stellar distribution in the Galaxy.

There has also been some discussion of a positional shift over the course of a microlensing event. Indeed, Alard et al. (1995; Alard 1996a) were able to measure astrometric shift in DUO \# 2, confirming that it was blended. However, since measurements were taken on a Schmidt plate, the shift was measured by using a center of gravity, rather than a PSF fit, and hence, the centroid position was only known to $\sim 0.2$ pixels at minimum light.

This technique has not been used more extensively, and what little attention that it has received has dwelt on the idea that if anything, astrometric shifts will be rare (Han 1997a). In this paper, we propose that astrometric shifts may be an extremely straightforward and robust method for searching for blends in microlensing events, and potentially even for 
constraining the blending parameter, $f$. As this is a relatively new technique, we shall try to deal with the problem in generality. That is, rather than concentrate on a particular survey or field, we shall use a simple (i.e. power law) luminosity functions, with known degrees of crowding. We think that this gives us a handle on the frequency and importance of astrometric shifts in a way that may be adapted to more realistic luminosity functions in a straightforward way.

The outline of the paper is as follows. In $\S 2$, we discuss the reasoning behind the expected astrometric shift, and show that this effect will be significant in crowded fields. In $\S 4$, we present a simulation of microlensing events with a power law luminosity function within artificial CCD frames. In $\S 3$, we present the results of those simulations, and in $\S 4$, we discuss the results, and suggest some future prospects.

\section{Motivation}

\subsection{Why do we expect an Astrometric Shift?}

Why do we expect to observe an astrometric shift in a blended microlensing event? Let us imagine that we only have a single, dark, lens which lenses one of two blended stars. For simplicity, we will assume that the PSF is a tophat function, and hence, the center of the best fit PSF (and the estimated center of light of the observed image) can be determined by a weighted average of the two components. If the lensed star has a fractional brightness, $f$, and is some distance $\Delta x_{0}$ from the center of light, then the companion contributes $(1-f)$, and is thus a distance $-\Delta x_{0} f /(1-f)$ from the center of light. If the lensed star increases in brightness by a factor, $A$, the center of light will shift to:

$$
\Delta x=\Delta x_{0} \frac{f(A-1)}{f(A-1)+1}=\Delta x_{0} \frac{A_{o b s}-1}{A_{o b s}},
$$

where $A_{o b s}$ is defined as above. This is illustrated in Figure 1. Though this relation assumes that there are only two contributing stars, in fact, this should hold for any number of stars, since we may put the stars into two groups: the lensed star, and all of the others. If we add the brightnesses and determine the centroid of the non-lensed stars, then this combined object should essentially contribute to the observed centroid like a single star.

One of the advantages of looking for an astrometric shift is that, unlike a color shift, it is does not depend critically upon the distribution of stars. Although the shape of the luminosity function and crowding may play a role in determining how many events are blended, the presence of a shift essentially only gives information about the relative geometry of the lensed source and the observed image, and thus requires no particular 
stellar population model. Thus it is not a statistical correction, and does not depend on unobservables.

Moreover, it is inexpensive. In current analysis of microlensing searches, a template catalog provides constant central positions for each of the observed objects, and the PSF for a given observation is determined from a set of fiducial stars (eg Alcock et al. 1996). While current methods may be ideal for identifying microlensing events, they are not directly sensitive to astrometric shifts. Therefore, we propose using the template catalogs as well as the frames containing the candidate events in order to do a PSF fit on the lensed stellar image. Since only a small fraction of the total frames will contain a lensed event, this is far less computationally taxing than performing PSF fitting throughout. However, such data is not yet available for any of the microlensing searches. The OGLE database will soon be available in the form of small subframes for the lensing candidates (Wozńiak \& Szymański 1997) and in a forthcoming paper, Goldberg \& Woźniak (1997) will look for shifts in the OGLE database.

Finally, even barring the possibility of finding a clear statistical relation between $f$ and $\Delta r_{0}$, (where $\Delta r_{0}^{2} \equiv \Delta x_{0}^{2}+\Delta y_{0}^{2}$ ), by performing a best fit to equation (6), we can determine the position of the lensed star with respect to the center of light. In this way, high resolution, ground-based followup observations can be used to observe the several stars comprising the light within the PSF. Since we can determine the position of the lensed star within that PSF, it becomes a simple matter to determine its fractional brightness compared to the observed PSF.

We suggest that it may be possible to detect a shift in a large percentage of microlensing events. Let us consider a case where there are exactly two stars within the PSF, one much brighter than the other. The PSF is very close to the center of the PSF. The other, however, may be anywhere within the PSF with little effect on the centroid position. If the dim object is lensed, we will expect to see a shift in the centroid. If the probability of finding the center of the dim star is distributed randomly within $1 R_{P S F}$, and the centroid position can be measured to an accuracy of $\sigma_{r}$, then the probability that there will be a measurable shift is $1-\left(\sigma_{r} / R_{P S F}\right)^{2}$, if $A_{o b s}$ is large. For reasonable values of $R_{P S F}=1$ pixel and $\sigma_{r}=0.1$ pixel, we expect that $\sim 99 \%$ of the events will have a measurable shift.

For an unblended (or very weakly blended star), however, we expect to see no shift, since the centroid of the observed star is defined by the center of the lensed star. 


\subsection{An Estimate: The Tophat Approximation}

Using a series of simplified assumptions, we can create a general order of magnitude technique for determining how often we expect to see astrometric shifts. The purpose of this exercise is twofold: First, this approximation is quick, and hence we can readily probe a family of luminosity functions. Second, we can use this approximation as a "sanity check" on the more rigorous simulations to follow. We would like to reassure ourselves that this effect is robust to our choice of parameters, and our choice of analysis software.

For the "Tophat Approximation", we assume a tophat PSF of some fixed radius (typically 3 "pixels", though the choice is arbitrary), and a power law luminosity function, $n(L) \propto L^{-\beta}$, where $\beta$ will be a free parameter. In fact, we will consider this as our flux distribution, since the lensed sources are approximately equidistant. For clarity, this distribution corresponds to a constant number per unit magnitude if $\beta=1$.

We use a power law luminosity function throughout. Though C. Alard (private communication) has suggested that a power law provides a poor fit in the galactic bulge (see eg Holtzman et al. 1993; Alard 1996b), it is not our purpose to provide a field specific estimate, but rather to suggest that astrometric shifts should be widely visible for a range of luminosity functions. Future work may wish to include more detailed models.

We model $N$ real stars per observed object (that is, blended within a given PSF or radius $R_{p s f}$ ), and we will set our detection threshold such that there are $M^{-1}$ observed objects per unit PSF area.

For each star within a sufficiently bright PSF, we assign a value of $f$ given by equation (11). We then convolve the distribution of $f$, with a probability function. That is, if a star with a given blending fraction, $f$, were lensed, what is the probability that $A_{o b s}>1.34\left(u_{\min }<1\right)$ ? This can be analytically determined and is not dependent upon our model, since from simple geometrical arguments, the probability distribution of $u_{\min }$ is expected to be uniform:

$$
P\left(A_{o b s}>1.34 \mid f\right)=\int_{1.34}^{\infty} p\left(A_{o b s} \mid f\right) d A_{o b s}=\sqrt{\frac{2 A_{\min }(f)}{\sqrt{A_{\min }^{2}(f)-1}}-2},
$$

where $A_{\min }(f) \equiv 1+0.34 / f$.

For each of the "observed" events, we can then determine a distribution of offsets from the centroid. Let us suppose that there is a group of stars with a known center of light and a known brightness, and let us further suppose that we then lay down an additional star, with a fractional brightness, $f$, randomly with respect to the initial group. It is simple to 
show that the probability that the additional (lensed) star will be placed a distance, $r^{\prime}$ from the initial centroid is simply $p\left(r^{\prime}\right) d r^{\prime}=2 r^{\prime} / R_{p s f}^{2} d r^{\prime}$. Thus, the distribution of the distance of the lensed star from the center of light, $\Delta r_{0}$, can be approximated as:

$$
p\left(\Delta r_{0}\right) d\left(\Delta r_{0}\right) \simeq \frac{2\left(\Delta r_{0}\right)}{(1-f)^{2} R_{p s f}^{2}} d\left(\Delta r_{0}\right) \quad ; \quad \Delta r_{0}<R_{p s f}(1-f) .
$$

This distribution is clearly correct in the limiting cases. If $f \ll 1$, we expect that the star can be virtually anywhere in the PSF, and by virtue of the larger area near the edges of the PSF, we will expect the lensed star to have a high value of $\Delta r_{0}$. If $f=1$, the star must be at the centroid.

Thus, we have a probability distribution of $f$ and a probability distribution of $\Delta r_{0}(f)$. We can relate these two, and in Figure 2 , we show a greyscale plot of the probability density of $p\left(\Delta r_{0}, f\right)$, for simple models with $N=10$ stars per PSF, $M=10$, and (a) $\beta=2$, (b) $\beta=3$.

In Figure 3, we show the expected distributions of $f$ and $\Delta r_{0}$ for observed microlensing events with $\beta=1.5,2$ and 3 . For the $\beta=2$ and $\beta=3$ case, these are integrations of Figure 2 over $\Delta r_{0}$ and $f$, respectively.

Note that for small values of $\beta$, the distribution is made up almost entirely of unblended $(f=1)$, and severely blended, $(f \ll 1)$, events. If events were distributed in this, photometric analysis could differentiate between the two (Woźniak \& Paczyński 1997). However, at higher $\beta$, there are a great many events at intermediate blending fraction. It is these cases in particular that we hope to probe in our simulation.

Using this estimate, we find that many events are expected to show a significant shift. For $\beta=1.5,2$, and 3 respectively, we find that in very crowded fields, $0.38,0.43$, and 0.89 of the events are expected to have shifts of $\geq 0.2$ pixels $(\sim 0.1 \times$ FWHM of the PSF $)$. These typically represent $\sim 0.68-0.89$ of the events with $f<0.9$.

\section{Method: A Simulation of Blended Events}

Thus far, we have given only an approximation of the blending in the case where we have perfect astrometry, no Poisson noise, an idealized PSF, and a complete catalog below some limiting magnitude. We now discuss a series of Monte Carlo simulations in which we try to take more physical constraints into account.

We first define a few terms, to which we refer throughout. We use the term "true" catalog to refer to the actual positions and brightnesses of stars which we use to generate a 
mock CCD image. The "observed" catalogs are the associated centers and brightnesses for observed objects computed by DAOphot package in IRAF (Stetson 1987). The "template" catalog is the collection of the most consistently identified objects over a series of many observations. Finally, a "lensed" catalog contains a collection of observed objects if we alter the brightness of one or more true star.

We first create a true catalog with a power law luminosity function. Each star is given a random position in coordinates of a $256 \times 256$ grid with the limits of floating point accuracy (corresponding to the dimensions of a CCD).

After creating a true catalog, we lay down a series of 15 observed images in order to construct a template catalog. First, the stars are laid down on a mock CCD frame with a Gaussian PSF of $\sigma \simeq 1.2$ pixels. Since there are random shifts between different observations of a field, a random offset of a fraction of a pixel is applied to all the stars before laying down a template image. A constant background of $\sim 2000$ counts per pixel (compared with a typical background of 500 - 1000 in the OGLE survey; Woźniak \& Szymański 1997) and Poisson noise are added, and all pixels above the saturation limit are truncated at that limit. We assume no read noise or bad pixels. The former should not be a major consideration since crowded fields are confusion, rather than photon limited, and the latter should effect all events democratically.

Each of the observed images are then analyzed using DAOphot, and in particular, brightnesses and centers of each star in each of the observed catalogs are computed using a PSF fitting routine. We assume that since there are a large number of stars in the frame, the width of the PSF is well known. We then cross compare each of the observed catalogs, and retain only those stars which appear in all 15 of the observed catalogs. The template catalog is the set of mean brightnesses, central positions and uncertainties, of these consistently observed stars.

We then proceed to compute a series of lensed catalogs. For a series of amplifications, we brighten each of the stars that appear within the PSF of a given observed object sequentially, and use DAOphot to create an observed catalog. For reasons of computational speed, we adjust the brightness of one true star in each of the template objects simultaneously. We then compare the lensed catalog to the template catalog to see whether each of the lensed objects is observed, and whether changing $A$ actually affects $A_{o b s}$. Since we have only the physical proximity of the true and observed star to suggest that they are related, the only way to test whether the two are observationally associated is to see whether varying the former also changes the latter.

For those true stars associated with an observed star, we create a series of mock 
microlensing events. We select a random $u_{\min }$ between zero and one for each star. If $A_{\text {max }}^{\text {obs }} \geq 1.34$ (corresponding to $u_{\min }=1$ for an unblended event), we include this event in our statistics.

Thus, for each event, we have a set of values of $A_{o b s}, \Delta x, \Delta y$, and $A$, where the former 3 are observables, and the latter is only known in a computational environment. Typically, we have $\sim 5-10$ measurements per event, about $\sim 0.2-0.5$ of the number in current microlensing searches.

We generate a mock light curves, by plotting $A_{o b s}$, versus $t$ (which can be computed from the known "true" magnification of the star). We also use our catalogs to compute $\Delta x_{0}$ and $\Delta y_{0}$, where $\Delta x_{0}$ is the best fit to equation (6) with a similar equation for $\Delta y_{0}$. By definition, $\Delta x_{0}$ and $\Delta y_{0}$ are equal to the position of the true lensed star with respect to centroid of the observed star at baseline. Finally, we determine the fraction of light in our lensed object, $f$, by doing a best fit to equation (2).

In Figure 4 , we show a typical parameter fit to a simulated microlensing event with $f_{\text {obs }}=0.018, \Delta r_{0}=2.3$ pixels, and $u_{\text {min }}=0.04$. This corresponds to an $A_{o b s} \simeq 1.4$. Note that in accordance with our initial assumptions, a strong blend is also accompanied by a large shift.

\section{Results}

In this section, we present the results of our simulations. We ran two simulations, each with 20000 stars. We used a power law luminosity function for both, one with $\beta=2$, and the other with $\beta=3$. The former contained $\sim 650$ template objects, and the latter contained $\sim 400$ objects. In both cases, about twice as many stars were typically observed, but many were rejected from the template catalog due to the fact that they were not observed in all of the catalogs. Since both fields were limited by crowding, a typical observed star was associated with $\sim 15$ blended true stars, down to about $1 \%$ of the observed threshold. This is consistent with the model used by Han (1997b). We verified that the observed template objects had a high correspondence to the brightest stars in the true catalog.

In order to compare the simulations to actual observational programs, we will briefly consider the crowding of the template catalog. The template catalogs contain $\sim 1$ star per 100 pixels. Though typical observed catalogs were about twice as crowded. By comparison, the OGLE survey (Udalski et al. 1992) has an average of $\sim 1 / 25$ pixel, while the MACHO LMC survey (Alcock et al. 1996a) has an average of $\sim 1$ monitored LMC star per 80 pixels. Future simulations may wish to model specific fields. 
After creating a set of lensed catalogs, and randomly selecting impact parameters, $u_{\min }$ for each of the consistently observed, lensed objects, we found $\simeq 500$ microlensing events in the $\beta=2$ simulation, and $\simeq 600$ events in the $\beta=3$ simulation.

In Figure 5, we show the results of these simulations. Panel (a) shows the distribution of $f$ (as fit to equation 2) against $\Delta r_{0}$ (as fit to equation 6) for the $\beta=2$ simulation. Panel (b) shows the distribution for the $\beta=3$ case. Note that the simplistic tophat approximation reproduces the gross features in the actual distribution of events (Figure 2), especially for $\beta=2$.

Some of the events are "measured" as having $f>1$. There are two reasons for this: First, the fits are based on measured data, and hence, any uncertainties in the data produce uncertainties in $f$. Within errors, many $f=1$ events can certainly be measured at $f>1$. Second, there is a significant background effect in crowded fields. Some of the light of the lensed star may be considered part of the background in the unlensed case, and hence, the increase in brightness (which can be determined differentially), can appear relatively more substantial than, in fact, it is. We shall consider these $f>1$ events to be unblended for the rest of the analysis.

Note also that the $\beta=2$ model has many unblended events with essentially no shift, while the $\beta=3$ model seems to have a larger fraction of events which have are severely blended. In the former case, we think of most observed stars being identified as a primary, and the many other contributing stars can only produce visible events by being in close proximity. On the other hand, in the $\beta=3$ case, the more democratic distribution of values of $f$ suggest that, due to the greater number of dim stars (from the steeper luminosity function), many observed "stars" contain no single true star which would be of sufficient brightness to be visible on its own.

In addition to the distribution of shifts and blending fractions, we are also interested in the relation between the two. Table 1 lists the number of events which were observed with significant shifts, compared with whether or not they were significantly blended. Note that in both simulations, $\sim 75 \%$ of significantly blended events had a large shift $\left(\Delta r_{0}>0.5\right.$ pixels $)$, while only $\sim 3 \%$ of unblended events had a large shift.

We consider the distribution of $f$ and $\Delta r_{0}$ and $u_{m i n}$, for each of the models, in order to see how often we expect to see shifts, or to estimate how often events will be significantly blended. Figure 6 a shows the distribution of values of $f$. Figure $6 \mathrm{~b}$ shows the distribution of $u_{\text {min }}$. It is strongly skewed to low values of $u_{\text {min }}$ due to events with small $f$, which will not be detected if the true value of $u_{\min }$ is close to unity. Figure Gc shows the distribution of shifts. 
Figure 6a is of considerable interest. In both models, there seem to be two populations of events. The first population consists of essentially unblended $(f \simeq 1)$ events, while the second group are highly blended $(f \ll 1)$, resulting in a bimodal distribution of blending fractions (with a typical FWHM of $\Delta f \sim 0.5$ ). In a sparse field the population of highly blended events would not be seen at all, since their relative importance is related directly to the number of stars below the observational threshold within the PSF of an observed image. This two component model may also describe a more realistic luminosity function.

In an actual observational program, we would only be able to measure Figure 6ic. However, even from this, we are able to make some estimates about the blending fraction. For example, we note that that about twice as many events have a small astrometric shift ( $\Delta r_{0}<0.5$ pixels), in the $\beta=2$ model as the $\beta=3$ model. To first order, one would think (correctly) that there will be about twice as many unblended events in the $\beta=2$ model as the $\beta=3$ model. Indeed, since the distribution of shifts (and with it, the distribution of $f$ ) is strongly dependent upon the luminosity function of stars below the detection threshold, this distribution may be a powerful probe of the stars in that regime. For a large survey like MACHO, with > 100 observed events, this distribution may actually be observed.

For a given scenario (Luminosity Function+Crowding+Detection Method) we can compute (numerically) the probability of observing an event with blending fraction, $f$, given some observed astrometric shift, $p\left(f \mid \Delta r_{0}\right)$, and likewise determine the probability distribution of astrometric shifts given some "true" blending fraction: $p\left(\Delta r_{0} \mid f_{0}\right)$. These probabilities are appropriately normalized vertical and horizontal traces through Figure 5 . Both dispersions are quite large. However, we may ask: if an event has a true blending fraction, $f_{0}$, what is the probability that we will estimate the blending fraction, $f$ ?

$$
P\left(f \mid f_{0}\right) d f=\int_{\Delta r_{0}=0}^{\infty} P\left(\Delta r_{0} \mid f_{0}\right) P\left(f \mid \Delta r_{0}\right) d\left(\Delta r_{0}\right) d f .
$$

Thus, we can calculate the expectation value, $f_{\text {meas }}$, and the associated dispersion, given some actual blending fraction, $f_{0}$.

In Figure 0, we present this result. The extreme cases of an unblended or a highly blended $(f \lesssim 0.1)$ event can be statistically distinguished from one another. However, moderate blends $(f \simeq 0.5)$ are generally consistent with either extreme.

\section{Discussion}




\subsection{Comparison with Previous Deblending Estimates}

Does this result constrain blending significantly? Woźniak \& Paczyński (1997) suggested that, in general, photometric information was insufficient to uniquely determine a value of $f$. For $f \gtrsim 0.08$, a significant fraction of events could not be distinguished from the unblended case at the $68 \%$ level. By comparison, from Figure 7 , we see that by using astrometric shifts, an event can be distinguished at the $68 \%$ confidence level if $f \leq 0.6$ for the $\beta=2$ model, and $f \leq 0.5$ for the $\beta=3$ model.

Regrettably, modest values of $f$ are difficult to uniquely identify using either astrometric shifts and light curve fitting, but in a slightly different way. Though essentially all significant shifts correspond to a significant blend, we cannot say that all significant blends will have a significant shift.

This method may also do quite well in comparison with the color shift method. First, searches for astrometric shift require no a priori model of color distribution. Moreover, a noticeable astrometric shift was predicted for $\gtrsim 0.6$ of the events in the $\beta=2$ model and $\gtrsim 0.8$ in the $\beta=3$ model. In both, those with small shifts ( $\leq 0.5$ pixels) were virtually all $(\geq 90 \%)$ unblended, and most of those with large shifts $(>0.5$ pixel) were severely blended. This can be contrasted with the $30 \%$ color shift detection rate suggested by Buchalter et al. (1996) for blending by the lens, and the $10 \%$ shift rate for background blends. They assumed $\sim 0.1$ bright $(V<19)$ stars per arcsecond in the bulge, and assuming 1 arcsecond seeing our distribution gives $\sim 0.06$ visible stars per arcsecond, suggesting that our degree of crowding is not unreasonable for current observations.

\subsection{Other Complications and Considerations}

We have used a fairly simple model of astrometric shift throughout. There may be considerable concern that we have used an overly simple luminosity function, or an unphysical degree of crowding. However, it was not our aim to deal with a specific survey or a specific field, and thus we have tried to treat the problem with generality. However in addition to these types of concerns, there are some additional complicating issues which we may wish to keep in mind.

The first of these is that we are not considering fixed positions for PSFs, and hence, the weight given to various background stars will vary over the course of a microlensing event. As a result of this, the value of $f$ will vary, and one might expect that equations (2) and (6) will no longer be strictly correct. In our simulations, this has not posed a problem. A constant value of $f$ throughout satisfies both relations adequately. It is less clear that 
a moving centroid will provide identical measured parameters as a constant centroid. By following the center of light, it may be that the amplification may be systematically higher than with a constant centroid owing to an increasing contribution of the lensed star.

Next, this method is not directly sensitive to blending by a binary companion since the angular separation between the two would be miniscule. Given that about $\sim 0.8$ of all bright primary stars are expected to have a companion (Abt et al. 1990), this is not a small effect. We can consider a worst case scenario. Imagine that all stars have a companion of equal brightness, but are assumed to be single stars. We will systematically overestimate $f$ by a factor of 2 . However, we will also underestimate the effective optical depth for a given Galactic model, since each monitored star will represent two chances to be lensed. In a more benign case in which one star is brighter than its companion, we will expect that there will be a bias toward observing the brighter star being lensed. Moreover, if it is significantly brighter than its companion, then the effect of blending (by the binary) will be small.

Finally, we have other observed parameters which we have not used directly. In attempting to deblend an event, we can also specify the observed brightness in our probability function. Consider a very bright object in a region with a steep luminosity function, for example. We may expect that a priori the star will be almost unblended, and thus, for a microlensing event which has a baseline level many $\sigma$ above the mean, we are virtually guaranteed that it is unblended. Looking at the brightest $10 \%$ of events in each of our simulations, we found that in the $\beta=3$ (brightest 48 events) only 4 events had a value of $f<0.8$, and of those, only 3 had a shift of greater than 0.5 pixels. For a flatter distribution like the $\beta=2$ simulation (brightest 56 events), the distribution of brightest events more strongly resembled the distribution of events as a whole, with $\sim 30$ events with $f<0.8$ and shift $>0.5$ pixels.

\subsection{Future Prospects}

We propose a twofold investigation into astrometric shifts of blended microlensing events, numerical and observational.

First, there is a need for more complex and survey specific simulations. We have used a pre-packaged (though commonly used) routine in order to determine centroid positions. More robust techniques exist. In particular DoPHOT (Schechter et al. 1995) has been extremely successful in determining positions in crowded fields. Likewise, a more accurate and field specific luminosity function, variable seeing, realistic distribution of observations, and so forth, may make it possible to calibrate a simulation with a particular observational 
program.

In conjunction with theoretical estimates, it is also hoped that researchers will begin to look for astrometric shifts within current observational programs. This can be done with existing data after the fact, and can be done extremely quickly if only a small subframe around the lensed object is considered. The OGLE data is being prepared in this form (Woźniak \& Szymański 1997) and Goldberg \& Woźniak (1997) have detected a shift of 0.7 pixels for OGLE \# 5, an event already considered to be blended.

More importantly, perhaps, is the fact that astrometric information gives us an unambiguous (and model independent!) determination of the centroid position of the actual lensed star compared to the observed centroid of the PSF. By performing high-resolution ground based or HST followup observations, the actual source star could be picked out of a very crowded field, and thus used to directly deblend the event.

The author would like to gratefully acknowledge the advice and many valuable discussions with Bohdan Paczyński, as well as helpful comments from Przemyslaw Woźniak, Christophe Alard, Michael Strauss, and Michael Richmond. This work was supported by NSF grant AST-9530478 and an NSF Graduate Research Fellowship.

\section{REFERENCES}

Abt, H. A., Gomez, A. E. , \& Levy, S. G., 1990, ApJS, 74, 551

Alard, C. 1996a, in Proc. IAU Symp. 173, Astrophysical Applications of Gravitational Lensing, (Eds: Kochanek, C. S. \& Hewitt, J. N., Dordrecht: Kluwer), p. 215

Alard, C. 1996b, A\& A, in press, astro-ph/9609165

Alard, C., Mao, S., \& Guibert, J. 1995, A\&A, 300, L17

Alcock, C. 1997, in "Unsolved Problems in Astrophysics," (Eds: Bahcall, J. N., \& Ostriker, J. P., Princeton University Press), p. 253

Alcock, C., et al. 1996, preprint, astro-ph/9606165

Bennett, D. P., et al. 1997, submitted to Nuc. Phys. B.

Buchalter, A., Kamionkowski, M., \& Rich, M. R. 1996, ApJ, 469, 676

Di Stefano, R., \& Esin, A. A. 1995, ApJ, 448, L1

Goldberg, D. M., \& Woźniak, P. 1997, in preparation

Han, C. 1997a, ApJ, 484, 555 
Han, C. 1997b, ApJ submitted, astro-ph/9704212

Holtzman, J. A. et al. 1993, AS, 106, 1826

Mao, S. \& Di Stefano, R. 1995, ApJ 440, 22

Paczyński, B. 1986, ApJ, 304, 1

Paczyński, B. 1996, ARA\& A, 34, 419

Pratt, M. R. et al. 1996, IAU Symp. 173, Astrophysical Applications of Gravitational Lensing (Eds: Kochanek, C. S. \& Hewitt, J. N., Dordrecht: Kluwer), p. 221

Schechter, P. L. Mateo, M. L., \& Saha, A. 1995m PASP, 105, 1342

Stetson, P. B., 1987, PASP, 99, 191

Udalski, A., Szymański, M., Kałużny. J., Kubiak, M., \& Mateo, M. 1992, Acta Astron., 42, 253

Udalski, A., Szymański, M., Kałużny. J., Kubiak, M., \& Mateo, M. 1993, Acta Astron., 43, 69

Udalski, A., Szymański, M., Mao, S., Di Stefano, R., Kałużny. J., Kubiak, M., Mateo, M., \& Krzemiński, W. 1994a, ApJ, 436, L103

Udalski, A., Szymański, M., Kałużny. J., Kubiak, M., Mateo, M., Krzemiński, W., \& Paczyński, B. 1994b, Acta Astron., 44, 227

Witt, H. J. \& Mao, S., 1995, ApJ, 447, L105

Woźniak, P. \& Paczyński, B. 1997, ApJ, in press

Woźniak \& Szymański 1997, in preparation 


\begin{tabular}{|c||c|c||c|c|}
\hline & \multicolumn{2}{|c|}{$\beta=2($ total $=485)$} & \multicolumn{2}{c|}{$\beta=3($ total $=495)$} \\
\hline \hline & $f>0.9$ & $f \leq 0.9$ & $f>0.9$ & $f \leq 0.9$ \\
\hline$\Delta r_{0}>0.5$ & 14 & 206 & 20 & 427 \\
\hline$\Delta r_{0} \leq 0.5$ & 188 & 77 & 71 & 77 \\
\hline
\end{tabular}

Table 1: A summary of the distribution of events in the $\beta=2$ and $\beta=3$ microlensing simulations. The events are roughly divided into "unblended" $(f>0.9)$, "significantly blended" $(f \leq 0.9)$, "unshifted" $\left(\Delta r_{0} \leq 0.5\right.$ pixels $)$, and "shifted" $\left(\Delta r_{0}>0.5\right.$ pixels $)$. 

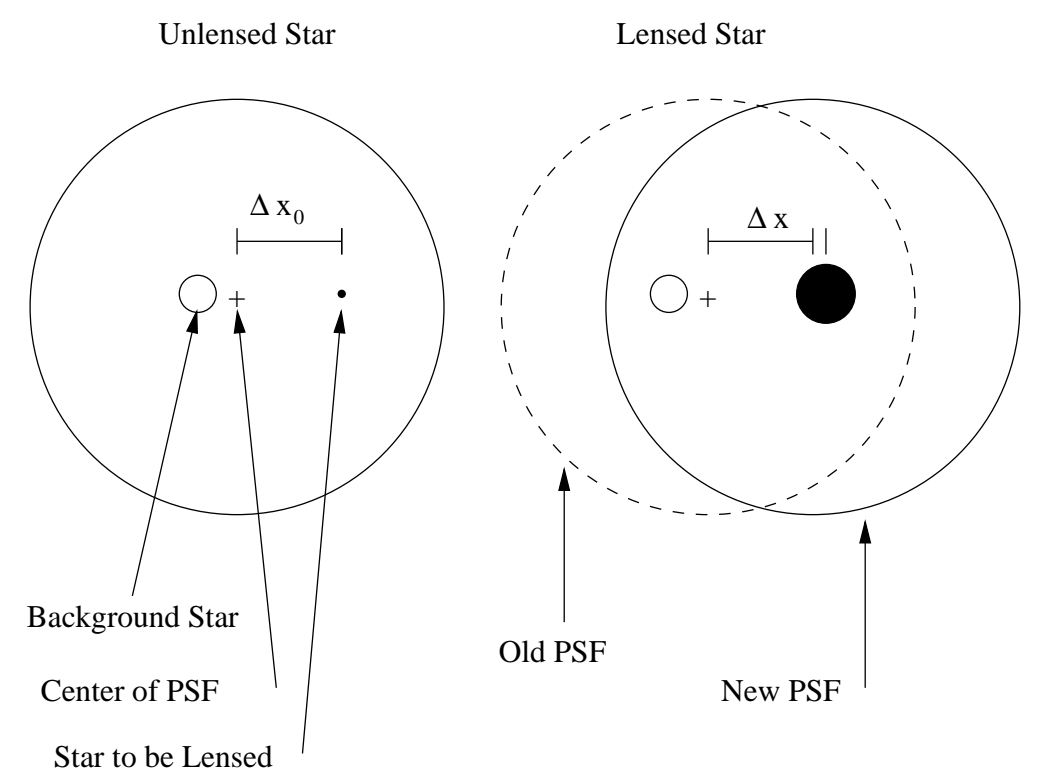

Fig. 1. - A schematic description of the shift of the center of light during a blended microlensing event. 

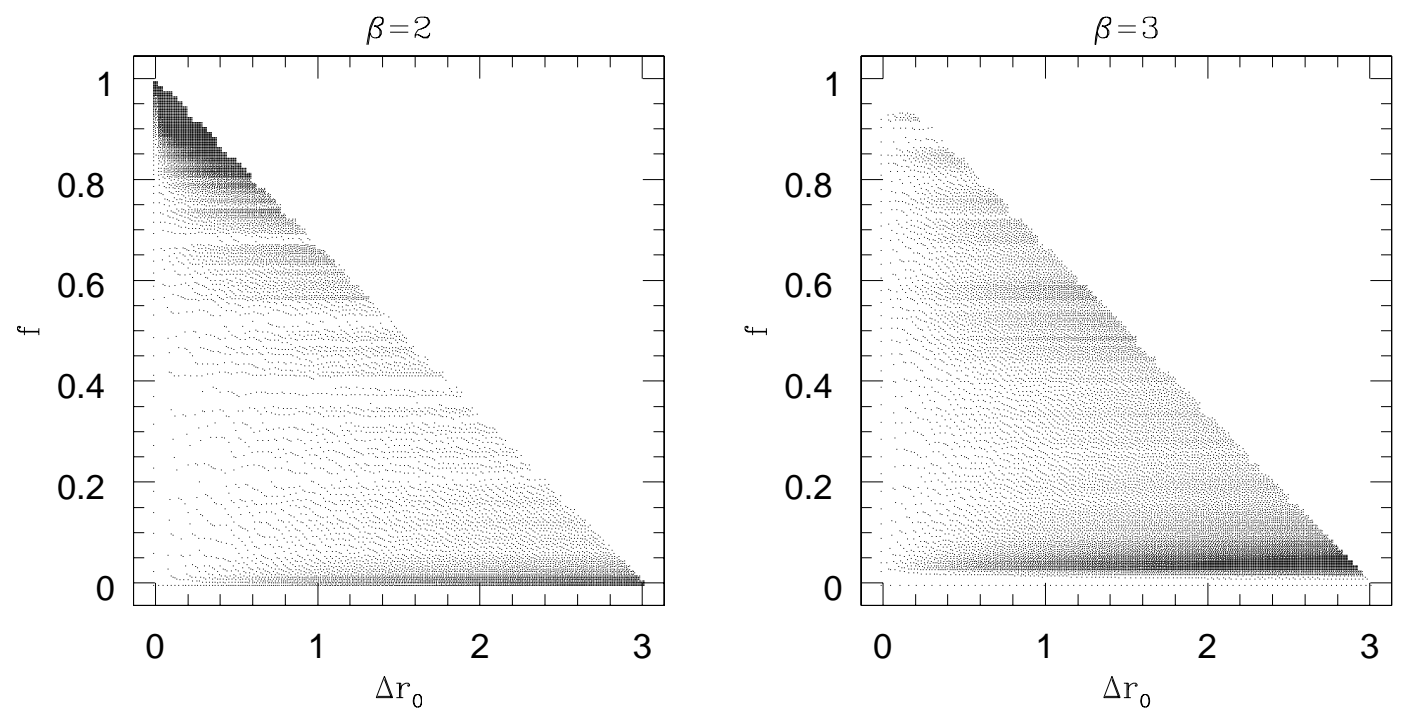

Fig. 2.- Covariant probability density of $f$ and $\Delta r_{0}$ for the $\beta=2$ and $\beta=3$ luminosity functions. 

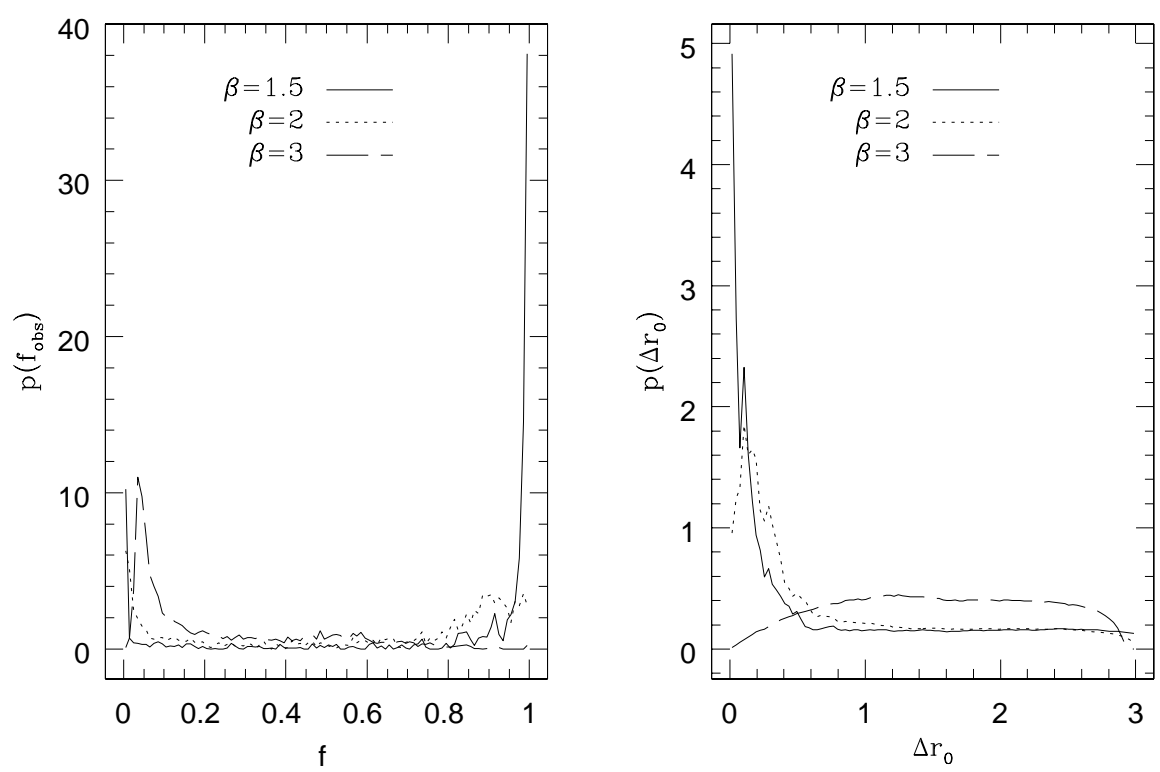

Fig. 3.- The expected distribution of $f$ and $\Delta r_{0}$ using the "Tophat Approximation" for a variety of luminosity functions. The luminosity functions are each power laws with $n(L) \propto L^{-\beta}$, where $\beta=1.5,2$, and 3 , and $N=10, M=10$ in all three. 

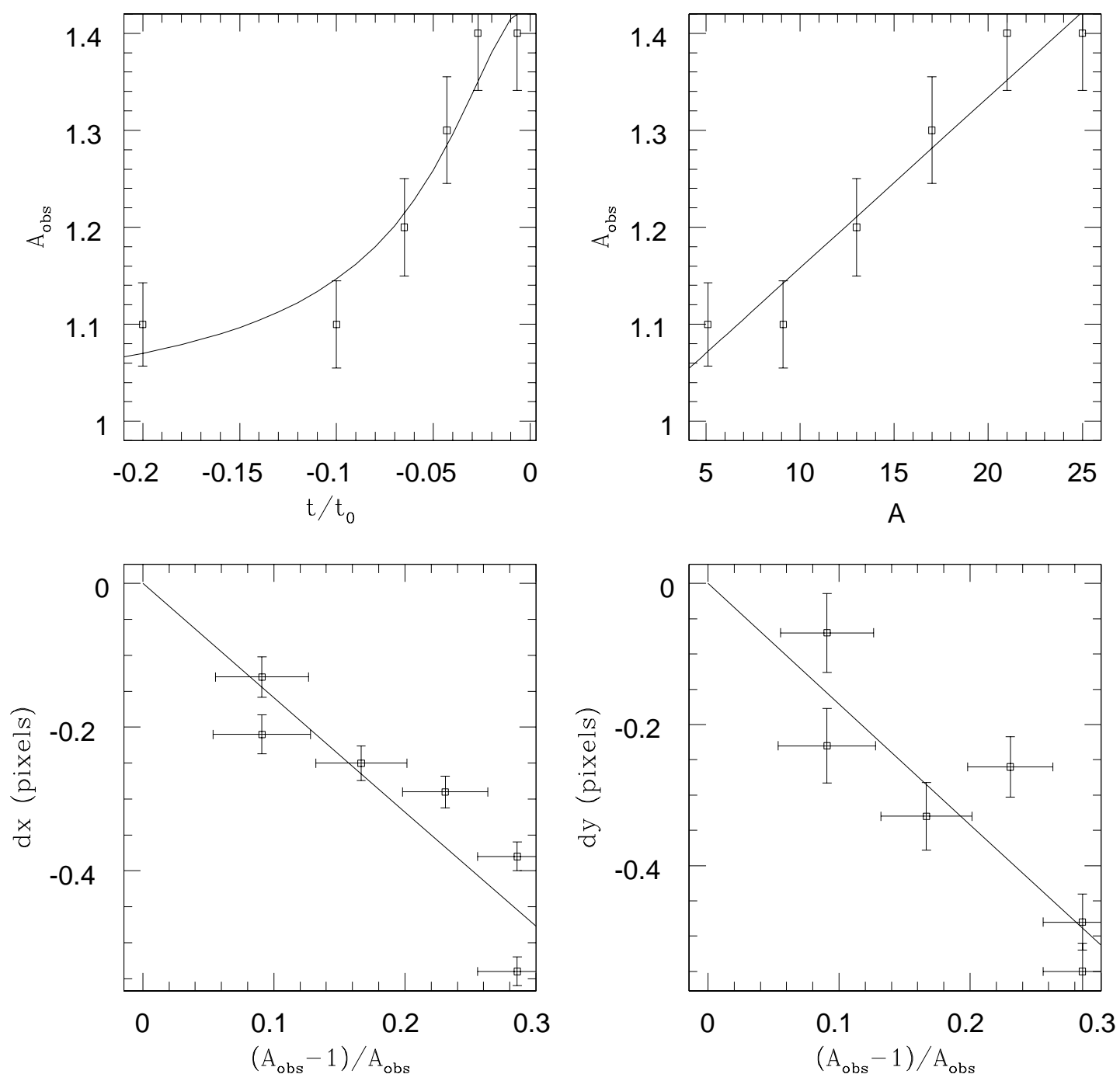

Fig. 4. - A mock microlensing curve, with $f=0.018$ and $u_{\min }=0.04$. (a) The photometric light curve with associated errors. The line is the curve associated with the microlensing parameters. (b)A plot of Magnification, $A_{o b s}$, versus Amplification, $A$. The line is a best fit linear relation given by equation (2). (c)The astrometric shift of the measured PSF centroid in the $x$ coordinate. The line is given by equation (6), and corresponds to an offset of $\Delta x_{0}=\simeq-1.6$ pixels. (d) Similar to panel c, but in the $y$ coordinate. The measured offset is $\Delta y_{0}=-1.7$ pixels. 

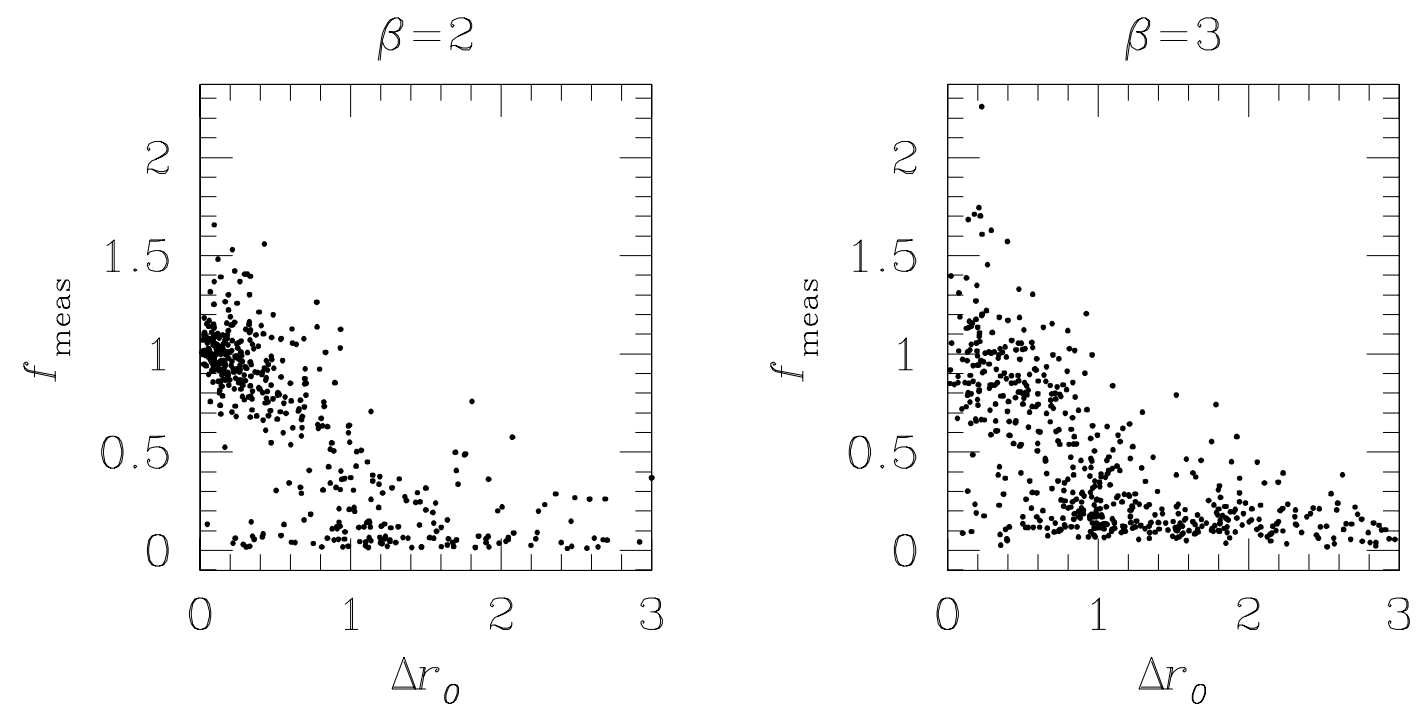

Fig. 5.- A scatter plot showing the relation between blending fraction, $f$, and measured offset, $\Delta r_{0}$ for the (a) $\beta=2$, and (b) $\beta=3$ case. 

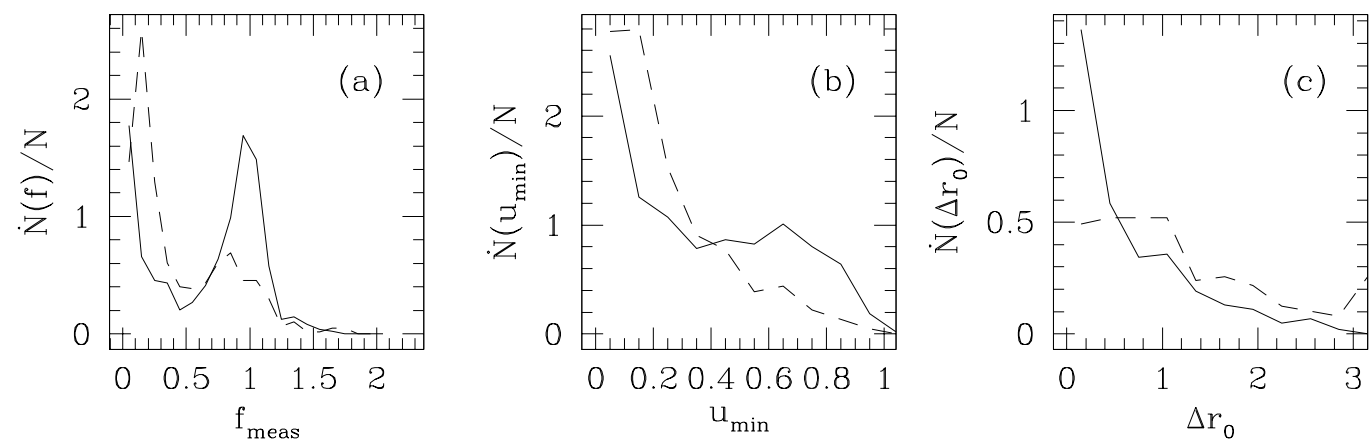

Fig. 6. - The results of the $\beta=2$ (solid) and $\beta=3$ (dashed) simulations. (a) The distribution of microlensing events as a function of $f$, binned into $\Delta f=0.2$ bins. (b) The distribution of microlensing events as a function of $u_{\text {min }}$, binned into $\Delta u_{\text {min }}=0.1$ bins. (c) The distribution of events as a function of astrometric shift, with data binned into $\Delta r_{0}=0.3$ pixel bins. 


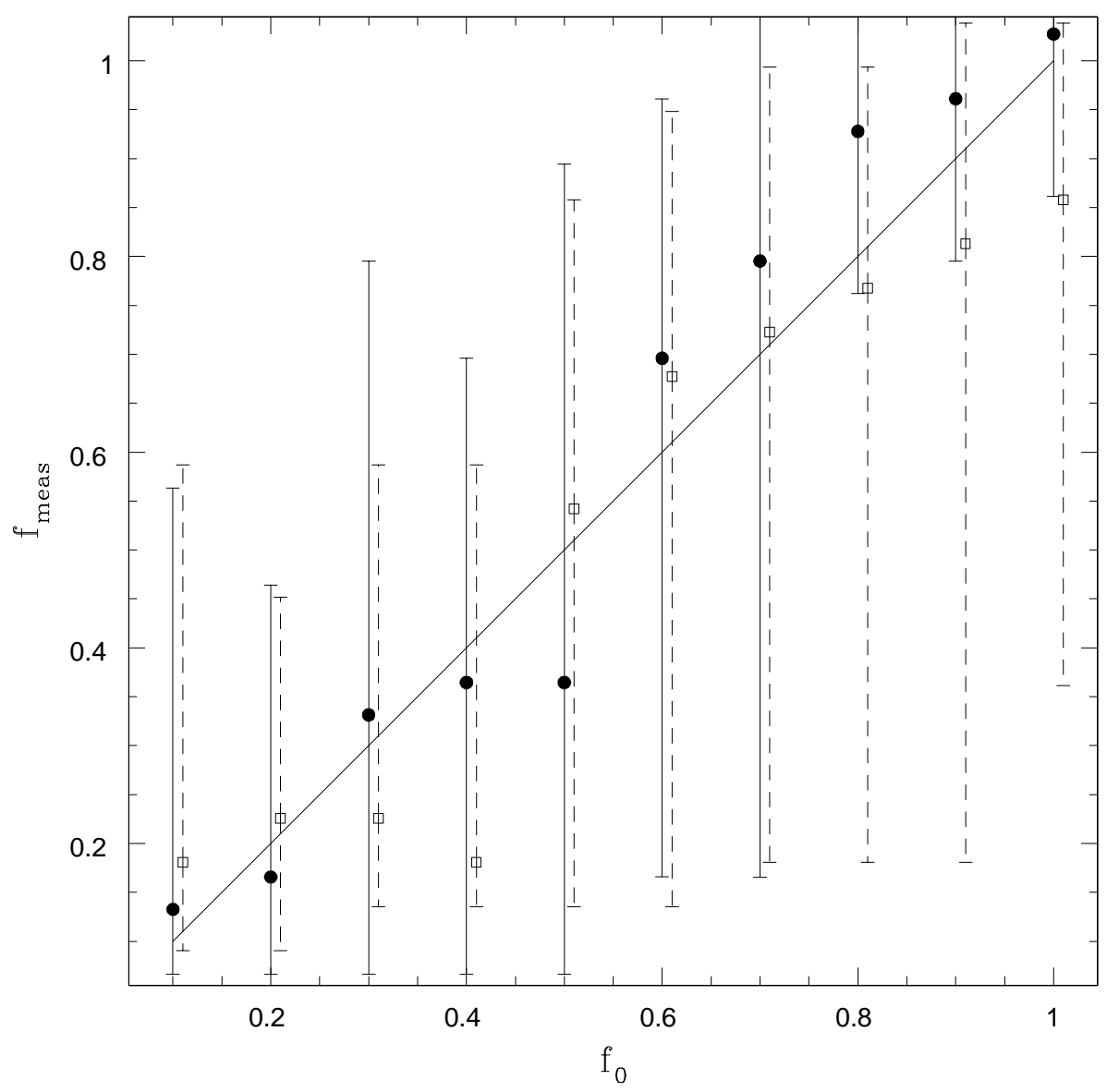

Fig. 7.- The distribution of actual and statistically measured values of $f$. The solid line shows an unbiased estimate of $f$. The solid circles and associated errors show the median and $1-\sigma$ dispersion of the estimated $f$ 's for the $\beta=2$ simulation, while the open squares show the probability distribution for the $\beta=3$ case 